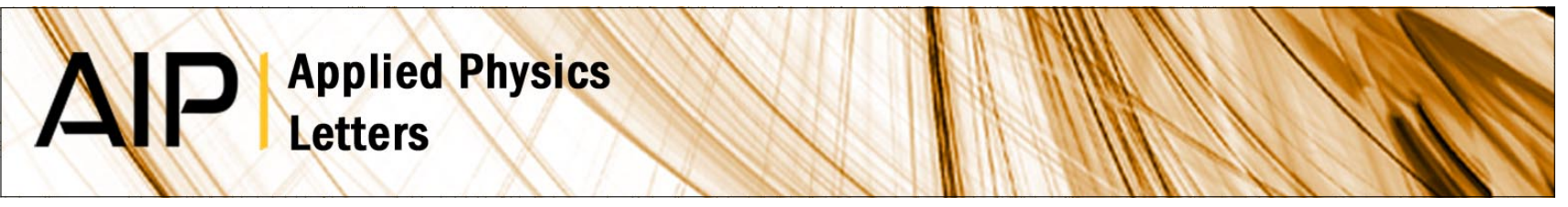

\title{
Nonlinear characterization of hydrogenated amorphous silicon waveguides and analysis of carrier dynamics
}

C. Lacava, P. Minzioni, E. Baldini, L. Tartara, J. M. Fedeli et al.

Citation: Appl. Phys. Lett. 103, 141103 (2013); doi: 10.1063/1.4823579

View online: http://dx.doi.org/10.1063/1.4823579

View Table of Contents: http://apl.aip.org/resource/1/APPLAB/v103/i14

Published by the AIP Publishing LLC.

Additional information on Appl. Phys. Lett.

Journal Homepage: http://apl.aip.org/

Journal Information: http://apl.aip.org/about/about_the_journal

Top downloads: http://apl.aip.org/features/most_downloaded

Information for Authors: http://apl.aip.org/authors

\section{ADVERTISEMENT}

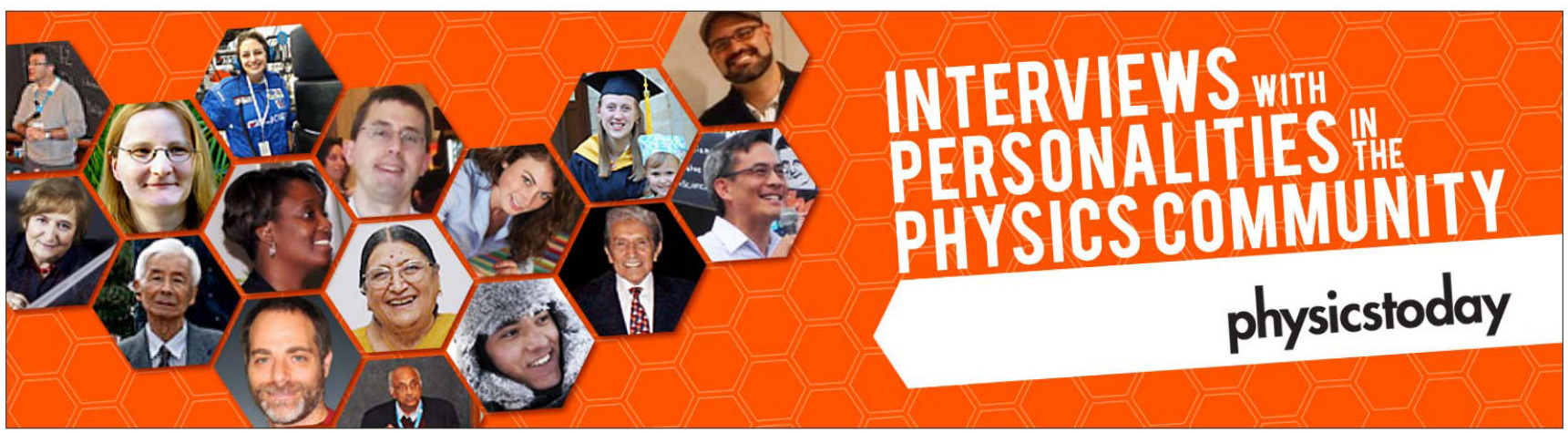




\title{
Nonlinear characterization of hydrogenated amorphous silicon waveguides and analysis of carrier dynamics
}

\author{
C. Lacava, ${ }^{1}$ P. Minzioni, ${ }^{1}$ E. Baldini, ${ }^{1}$ L. Tartara,${ }^{1}$ J. M. Fedeli, ${ }^{2}$ and I. Cristiani ${ }^{1}$ \\ ${ }^{1}$ Department of Electrical, Computer, and Biomedical Engineering, and CNISM, University of Pavia, \\ 27100 Pavia, Italy \\ ${ }^{2}$ CEA-Leti MINATEC Campus, 38054 Grenoble Cedex 9, France
}

(Received 29 July 2013; accepted 14 September 2013; published online 30 September 2013)

\begin{abstract}
In this paper, we determine the optical nonlinear coefficient of hydrogenated amorphous silicon (a-Si:H) waveguides. Up to date, the data reported in the scientific literature for similar structures show a very large variability and the final assessment of their nonlinear performance is still an open issue. We performed a complete and careful characterization of more than 50 waveguides. A nonlinear coefficient of $790+\mathrm{j} 20 \mathrm{~W}^{-1} \mathrm{~m}^{-1}$ was found, confirming that a-Si:H is a good candidate for nonlinear silicon photonic devices. Nevertheless, free-carrier-dynamics exhibits a recombination time in the nanosecond range, which can hinder their exploitation in ultrafast applications requiring high-power optical beams. (C) 2013 AIP Publishing LLC. [http://dx.doi.org/10.1063/1.4823579]
\end{abstract}

Hydrogenated amorphous silicon (a-Si:H) is a promising material for silicon photonics. The low temperature required for its deposition provides an excellent compatibility with standard CMOS (complementary metal-oxide-semiconductor) processes. Besides, recent works pointed out that a-Si:H exhibits a large optical third order nonlinearity (larger than that of crystalline silicon) ${ }^{1}$ making this material an ideal candidate for the development of silicon-based all-optical signal processing devices. At the state of the art, the data reported in the literature, concerning a-Si:H nonlinear parameters, show a great disagreement. ${ }^{1-7}$ As shown in Fig. 1, the real part of the nonlinear coefficient $\gamma$, measured in waveguides with similar mode field diameter and linear properties, shows a great variance, covering the whole range from $100 \mathrm{~W}^{-1} \mathrm{~m}^{-1}$ up to $3000 \mathrm{~W}^{-1} \mathrm{~m}^{-1}$. In addition, a similar spread in the data concerns also the two photon absorption (TPA) coefficient of the material and the free carrier lifetime, both having a considerable impact in ultrafast applications and in the design of nonlinear devices. The origin of such a great disagreement among the data reported in the literature is still an open issue. The different a-Si layer deposition process $^{1,2,5}$ could have a crucial role.

In this paper, we present the results achieved by an accurate nonlinear characterization of more than $50 \mathrm{a}-\mathrm{Si}: \mathrm{H}$ waveguides fabricated by CEA-Leti laboratories. The data set reported in this work was obtained by characterizing several dices, each containing optical waveguides with lengths ranging from 0.2 to $8 \mathrm{~cm}$, thus, assuring a high level of reliability of the measured data.

The obtained results demonstrate that a-Si:H exhibits an enhanced figure of merit $\left(\mathrm{FOM}^{8}\right)$ with respect to standard crystalline silicon, even if the TPA and Free Carrier Absorption (FCA) effects are not negligible, when very high beam intensities are considered, thus, imposing some limitations for ultrafast applications.

To fabricate the waveguides, a 220-nm thick film of a-Si:H film was deposited by means of Plasma Enhanced Chemical Vapor Deposition (PECVD) at $350^{\circ} \mathrm{C}$, on a $1.7-\mu \mathrm{m}$ thick silicon-oxide layer. A first etching step was used to define grating coupler patterns with an etching depth of
$70 \mathrm{~nm}$. After deposition of a silica hard mask, a full etch step (etching depth of $220 \mathrm{~nm}$ ) was performed to define the waveguide layout. Several straight and serpentine waveguides (with a fixed $500 \times 220 \mathrm{~nm}$ cross section) were obtained with lengths varying from $0.26 \mathrm{~cm}$ to $8.26 \mathrm{~cm}$. Finally, a protective $500 \mathrm{~nm}$ oxide upper cladding was deposited on the top of the waveguides. Focusing grating couplers, designed to only couple the transversal electric mode (TE-mode), were fabricated at each side of each waveguide.

As a first step, we performed a linear characterization of the waveguides, which is a fundamental step towards the accurate evaluation of all the nonlinear parameters. The transmission loss was experimentally evaluated by combining several measurements on waveguides with different length. Coupling efficiency was optimized by setting the angle between the axis perpendicular to the grating surface and the input single mode fiber (SMF) at $12^{\circ}$. The grating coupler exhibited a $-3 \mathrm{~dB}$ transmission bandwidth equal to $33 \mathrm{~nm}$, with a central wavelength of $1550 \mathrm{~nm}$. An all fiber polarization controller was used in order to set the polarization state of input beam. The input power was monitored by a 99\%-1\% fiber-based tap coupler, located at the input side. At the waveguide output, the light was coupled to another single-mode fiber tilted by the same angle via a second

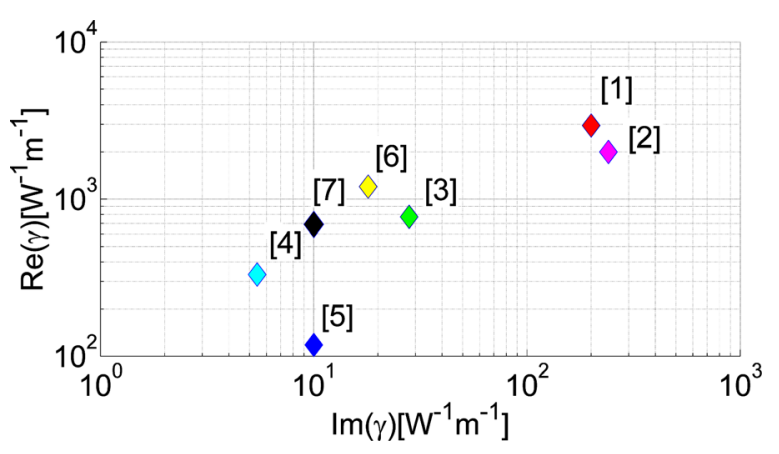

FIG. 1. Diagram showing the values of the real and imaginary part of $\gamma$ reported in the scientific literature, for waveguides similar to those analyzed in this paper. Given the very large difference in the measured values, a log$\log$ plot was used. 
TABLE I. Linear Loss coefficients obtained from the linear characterization of more than 50 a-Si:H waveguides.

\begin{tabular}{llcc}
\hline \hline Parameter & Value & Standard deviation & Unit \\
\hline$\alpha_{C O U P}$ & 9.9 & 0.1 & $\mathrm{~dB} /$ grating \\
$\alpha_{0}$ & 4.2 & 0.4 & $\mathrm{~dB} / \mathrm{cm}$ \\
$\alpha_{B N D}$ & 0.020 & 0.005 & $\mathrm{~dB} / 90^{\circ}$-bend \\
\hline
\end{tabular}

grating and a power meter was then used to monitor the output power. The overall loss was attributed to three different contributions: fiber-to-chip coupling $\left(\alpha_{\text {COUP }}\right)$, measured at the center of grating band, propagation in the straightwaveguide section $\left(\alpha_{0}\right)$, and waveguide bends $\left(\alpha_{B N D}\right)$. Thanks to the presence of waveguides with different lengths $(L)$ and with a different number $(N)$ of $90^{\circ}$-bends (always with $10 \mu \mathrm{m}$ curvature radius), we separately assessed the loss introduced by each element. The obtained results, reported in Table I, show that the standard deviation of measured parameters is about $10 \%$. Propagation losses are comparable to those reported in Refs. 1-4, and 6. Significant lower values could be achieved by means of low roughness resist and etching as shown in Ref. 5. Moreover, we note that the coupling loss resulted significantly larger than expected ( $\approx 9.97 \mathrm{~dB} /$ grating), probably due to an over-etching error occurred in grating fabrication.

After the analysis of linear properties, the nonlinear parameters of the waveguides were tested, starting with the assessment of the real part of the $\gamma$ coefficient, $\operatorname{Re}(\gamma)$, by means of a continuous wave (CW) Four Wave Mixing (FWM) experiment, realized using the setup shown in Fig. 2 (left). An intense CW beam (the FWM "pump") and a weaker CW-radiation ("signal") tunable in the range of 1530-1560 nm were combined by a 90/10 (pump/signal) fiber coupler and input to the waveguide under test. All-fiber polarization controllers were used to set the input polarization state of both radiations. The output radiation was collected by a SMF, coupled to an Optical Spectrum Analyzer (OSA), and the FWM efficiency was evaluated as the ratio between the power of the idler beam and the signal beam, both measured at the waveguide output. As an example, we report in Fig. 2 (right) the optical spectrum measured at the output of a 2.26-cm long spiral waveguide, and we show the corresponding FWM efficiency. The pump-to-signal detuning was initially set to a very small value $(\approx 0.4 \mathrm{~nm})$, to make negligible the impact of dispersion-induced phase-mismatch. In addition, all the measurements were performed in a "moderate-power" regime (power in the waveguide $<10 \mathrm{dBm}$ ), so that TPA and FCA effects could be considered as negligible.

Starting from the experimental data, $\operatorname{Re}(\gamma)$ was then calculated using Eq. (1), where $L_{\text {eff }}$ is the effective nonlinear waveguide length, ${ }^{9}$ and the $\eta$ coefficient accounts for both propagation loss and the phase-mismatch induced by waveguide dispersion ${ }^{9}$

$$
\operatorname{Re}(\gamma)=\frac{\sqrt{P_{i}(L) / P_{s}(L)}}{\eta P_{p}(0) L_{e f f}} .
$$

Waveguides with different lengths (in the range of $0.26-4.68 \mathrm{~cm}$ ) fabricated on several (50) dices were measured and the pump power was varied from -20 to $5 \mathrm{dBm}$ (at the beginning of the strip waveguide). We estimated $\operatorname{Re}(\gamma)=790 \pm 50 \mathrm{~W}^{-1} \mathrm{~m}^{-1}$ with an error that can be mainly ascribed to the coupling loss uncertainty.

It is worth underlining that the obtained value is in good agreement with the results reported for similar waveguides in one work only. ${ }^{3}$ Assuming the mode effective area equal to $\approx 0.14 \mu \mathrm{m}^{2}$ as resulted from numerical simulations, we could estimate that the nonlinear refractive index $\mathrm{n}_{2}$ of a-Si:H is $\approx 2.8 \times 10^{-13} \mathrm{~cm}^{2} / \mathrm{W}$. Moreover, it is important to highlight that we measured FWM efficiency also as a function of the time exposure, and differently from what reported in Ref. 3, we never observed any reduction of the nonlinear efficiency, even after propagation of high-power $\mathrm{CW}$ radiation $(>20 \mathrm{dBm})$ in the waveguides for a time-interval $>4 \mathrm{~h}$.

By measuring the FWM efficiency as a function of the pump-to-signal detuning $(\Delta \lambda)$, we could estimate the value of waveguide dispersion at $\lambda=1550 \mathrm{~nm}$, which resulted $\approx 380 \mathrm{ps} \mathrm{nm}^{-1} \mathrm{~km}^{-1}$. As no modulation-instability peaks were observed in the FWM-efficiency vs. detuning curve, we expect the waveguide to be in the normal-dispersion regime. 9

As a second step, the imaginary part of the nonlinear coefficient, $\operatorname{Im}(\gamma)$, was obtained by measuring the transmission of short pulses as a function of the input power in a propagation regime involving TPA. The exploitation of $120 \mathrm{fs}$ pulses at $82.5 \mathrm{MHz}$ repetition rate (duty-cycle $\approx 10^{-5}$, output by a Spectra Physics OPAL tuned at $1550 \mathrm{~nm}$ ) allowed distinguishing the impact of TPA from that of FCA. The last one mainly depends on the average power, and the pulse-to-pulse time interval is sufficiently large to allow for carriers recombination. The value of $\operatorname{Im}(\gamma)$ was then calculated starting from the following differential equation, ${ }^{8}$ where $P(z)$ is the pump power and $\alpha_{0}$ is the linear loss:

$$
\frac{d}{d z} P(z)=-\alpha_{0} P(z)-2|\operatorname{Im}(\gamma)| P^{2}(z)
$$

By assuming a hyperbolic-secant pulse temporal-profile, it is possible to analytically derive the dependence of the average transmitted power, $P_{T}$, on the input pulse peak power $P_{0}$ (Ref. 8)
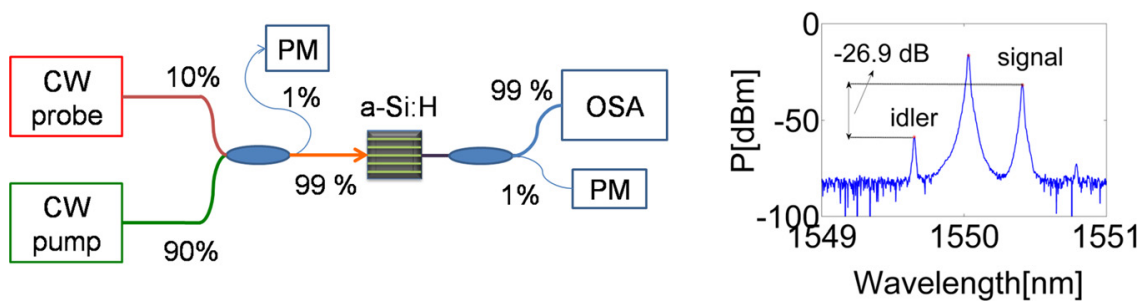

FIG. 2. On the left: FWM experimental scheme $(\mathrm{PM}=$ Power Meter. OSA = Optical Spectrum Analyzer). On the right: example of a trace recorded by the OSA. 
$P_{T}=e^{-\alpha_{0} L} \frac{\ln \left(\sqrt{2|\operatorname{Im}(\gamma)| L_{e f f} P_{0}}+\sqrt{1+2|\operatorname{Im}(\gamma)| L_{e f f} P_{0}}\right)}{\sqrt{\left(2|\operatorname{Im}(\gamma)| L_{e f f} P_{0}\right)\left(1+2|\operatorname{Im}(\gamma)| L_{e f f} P_{0}\right)}} P_{0}$

Using the $\alpha_{0}$ previously evaluated and the pulse peak power that can be extracted from the experimental data, we could thus assess the value of $\operatorname{Im}(\gamma)$ by fitting the $P_{T}$-vs- $P_{0}$ curve. In Fig. 3 , we show three typical $P_{T}$-Vs- $P_{0}$ curves, obtained on waveguides with different lengths, all well fitted by using the same value for $\operatorname{Im}(\gamma)=20.5 \mathrm{~W}^{-1} \mathrm{~m}^{-1}$ with a relative error of $\pm 5 \%$.

By combining the data on real and imaginary part of $\gamma$, one can easily estimate a $\mathrm{FOM}=3.06$, confirming that $\mathrm{a}-\mathrm{Si} \mathrm{H}$ is a very promising material for the realization of nonlinear all-optical devices. These results push for a proper characterization also of the phenomena related to FCA, which could indeed limit the maximum operation speed of an a-Si:H based device operated in high power regime. Also, in this respect, the data reported in the scientific literature show strong discrepancies with free-carriers recombination time-constants ranging from values $<100$ fs (Ref. 5) up to a few nanoseconds. ${ }^{3}$

The impact on pulse propagation of the FCA can be described by the following equation, where $\alpha_{F C}$ is the absorption coefficient due to free carriers, and $N_{F C}$ is the number of free carriers per unit of volume:

$$
\frac{d}{d z} I(z, t)=-\left[\alpha_{0}+\alpha_{F C} N_{F C}(t)\right] I(z, t)-\beta I^{2}(z, t) .
$$

The value of $\alpha_{F C}$ and the recombination time of free carriers generated by TPA were separately determined by performing two different experiments, both exploiting TPA to excite free carriers.

The first experiment, aimed at the evaluation of free carriers' recombination time, was carried out by using a pulsed pump beam (120 fs optical pulses, output by an OPAL Spectra Physics) to excite FC, and a CW probe beam to measure the temporal behavior of the transmission coefficient. The two beams were tuned at $\lambda_{\mathrm{P}}=1540 \mathrm{~nm}$ and $\lambda_{\mathrm{S}}=1560 \mathrm{~nm}$, respectively, combined by a $50 / 50$ fiber coupler and then input to the waveguide. At the waveguide output, the pump beam was removed by using two band-pass

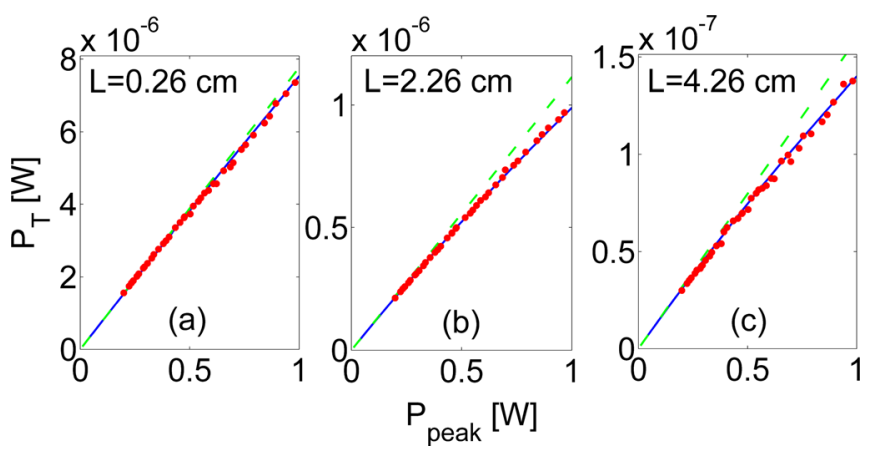

FIG. 3. $\mathrm{P}_{\mathrm{T}}$-Vs- $\mathrm{P}_{0}$ curve for (a) $0.26 \mathrm{~cm}$, (b) $2.26 \mathrm{~cm}$, (c) $4.26 \mathrm{~cm}$ long waveguides. Red circles are experimental data while the blue trace is the fitting function. The green-dashed line represents the theoretical linear transmission without TPA effect. filters, which ensured an extinction ratio of $50 \mathrm{~dB}$, and the probe beam was detected through a fast oscilloscope (HP $15 \mathrm{GHz}$ model 11982A) to retrieve the temporal behaviour. For low values of pump peak power (e.g., $P_{0}<10 \mathrm{dBm}$ in the waveguide), the transmission of the probe was almost constant in time; conversely, for $P_{0}>10 \mathrm{dBm}$, a periodic depletion of the probe beam was observed, in correspondence of pump beam pulses, as shown in Fig. 4 (left panel) which reports the experimental trace obtained with a $0.26 \mathrm{~cm}$-long waveguide. This behavior is related to the abrupt rise of $N_{F C}$ induced by TPA that can be described through the following equation: ${ }^{10}$

$$
\frac{d}{d t} N_{F C}(z, t)=\frac{\beta I^{2}(z, t)}{2 h \omega_{0}}-\frac{N_{F C}(z, t)}{\tau} .
$$

After the initial power drop, caused by the TPA-excited freecarriers, a two-phase recovery process can be observed: a very fast process responsible for the initial recovery of about $10 \%$ of transmission and a second (slower) process yielding a relatively long power-recovery tail. Experimental data can be fitted by the superposition of two exponential functions with time $\tau_{1}<50 \mathrm{ps}$ (fast recovery) and $\tau_{2}=1.87 \mathrm{~ns}$. Due to the limited bandwidth of the photodiode, we were not able to assess the fast temporal dynamics of the first exponential tail that can be considered just as an upper bound value.

We ascribe the initial fast response to the carrier thermalization in the extended band tails as analogously reported in Ref. 11. In principle, for higher pump power regimes, also cross-TPA could contribute to the initial fast response, and further studies are currently ongoing to clarify this point. On the other hand, the slower transient, responsible for the largest fraction of the FCA, can be associated to the excited carriers that diffuse and recombine at the waveguide surfaces. We note that this slow FCA term is quite large, in contrast with the results reported in Ref. 5, and thus it can strongly affect the ultrafast behavior of optical devices operated at high input power.

In the second experiment, carriers were excited using a $\mathrm{CW}$ beam, so that the time evolution of $N_{F C}$ can be described by Eq. (5). It is thus possible to evaluate $N_{F C}$ in steady state conditions and to substitute the obtained value in Eq. (4), removing also the time-dependence of $I$, as a $\mathrm{CW}$ beam is considered

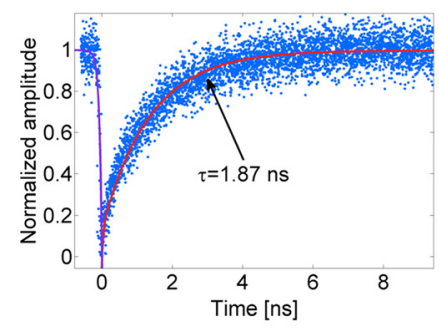

(a)

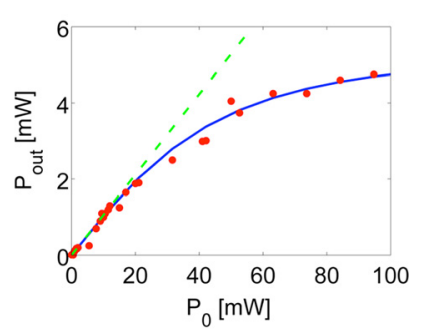

(b)
FIG. 4. Left: Free carrier recovering time experimental trace $\left(\mathrm{P}_{0}=10 \mathrm{~W}\right)$. Right: example of the transmission curve used to estimate $\alpha_{F C}$ $(\mathrm{L}=2.26 \mathrm{~cm})$; red circles are the experimental data, the blue trace is the fitting function, and the green dashed line represents the theoretical curve without TPA and FCA effects. 


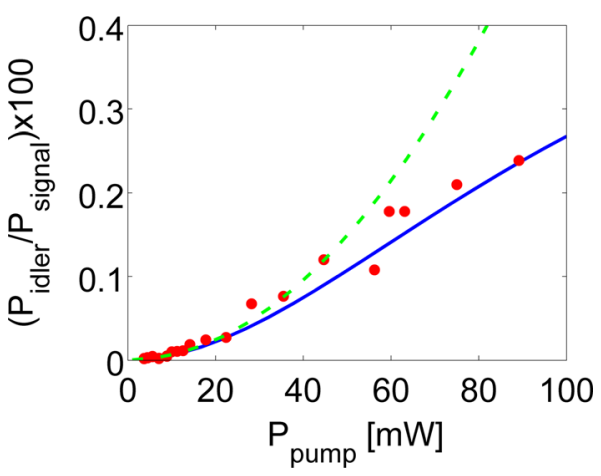

FIG. 5. Four wave mixing efficiency as a function of pump power $(\mathrm{L}=2.26 \mathrm{~cm})$. Red circles are the experimental data, the blue trace is the fitting function, and the green dashed line represents the theoretical curve without TPA and FCA effects.

$$
\frac{d}{d z} I(z)=-\alpha_{0} I(z)-\beta I^{2}(z)-\alpha_{F C} \frac{\beta \tau}{2 h \omega_{0}} I^{3}(z) .
$$

By exploiting $\alpha_{0}, \beta$, and $\tau$ parameters that were previously determined, $\alpha_{F C}$ could be experimentally evaluated by measuring the output power as a function of input power, and by fitting the obtained curve by using Eq. (6) (see Figure 4, right). Using this procedure, we obtained an $\alpha_{F C}$ value equal to $0.8 \times 10^{-17} \mathrm{~cm}^{2}$, which is only slightly lower than that measured in c-Si waveguides. ${ }^{12}$

As a final validation of the obtained parameters, we repeated the FWM experiments by measuring the conversion efficiency for larger pump powers, in a regime where the role of TPA and FCA becomes crucial, limiting the maximum achievable efficiency. In Fig. 5, we report the conversion efficiency-vs-pump input power: red circles represent the experimental values, while the blue line is obtained by numerical simulations, considering the above reported values of $\alpha_{0}, \beta, \tau$, and $\alpha_{F C}$. The dashed green line represents the predicted FWM efficiency when TPA and FCA are not present.

The very good agreement between the experimental results and the values calculated by numerical simulations confirms the reliability of our previous analysis. We can thus conclude that a-Si:H is a very promising material for the realization of nonlinear optical devices by CMOS-compatible technologies. Furthermore, the results obtained for the $\gamma$ coefficient are in good agreement with those reported in Ref. 3, but at contrast no degradation of the material properties, even after long-time operation with high beam power, was observed.

The authors acknowledge the staff team of CEA-Leti clean rooms for the waveguides fabrication.

${ }^{1}$ K. Wang and A. Foster, Opt. Lett. 37, 1331 (2012).

${ }^{2}$ K. Narayanan and S. F. Preble, Opt. Express 18, 8998 (2010).

${ }^{3}$ B. Kuyken, H. Ji, S. Clemmen, S. Selvaraja, H. Hu, M. Pu, M. Galili, P. Jeppesen, G. Morthier, S. Massar, L. Oxenløwe, G. Roelkens, and R. Baets, Opt. Express 19, B146 (2011).

${ }^{4}$ J. Matres, G. Ballesteros, P. Gautier, J. Fédéli, J. Martí, and C. Oton, Opt. Express 21, 3932 (2013).

${ }^{5}$ S. Suda, K. Tanizawa, Y. Sakakibara, T. Kamei, K. Nakanishi, E. Itoga, T. Ogasawara, R. Takei, H. Kawashima, S. Namiki, M. Mori, T. Hasama, and H. Ishikawa, Opt. Lett. 37, 1382 (2012).

${ }^{6}$ C. Grillet, L. Carletti, C. Monat, P. Grosse, B. Ben Bakir, S. Menezo, J. Fedeli, and D. Moss, Opt. Express 20, 22609 (2012).

${ }^{7}$ L. Carletti, C. Grillet, P. Grosse, B. Ben Bakir, S. Menezo, J. Fédéli, D. J. Moss, and C. Monat, in Proc. SPIE 8767, 876706 (2013).

${ }^{8}$ J. Matres, C. Lacava, G. Ballesteros, P. Minzioni, I. Cristiani, J. Fédéli, J. Martí, and C. Oton, Opt. Express 20, 23838 (2012).

${ }^{9}$ G. P. Agarwal, Nonlinear Fiber Optics, 2nd ed. (Academic Press, New York, 1995).

${ }^{10}$ A. Trita, I. Cristiani, V. Degiorgio, D. Chrastina, and H. von Kanel, Appl. Phys. Lett. 91, 041112 (2007).

${ }^{11}$ K. Narayanan, A. Elshaari, and S. Preble, Opt. Express 18, 9809 (2010).

${ }^{12}$ Q. Lin, O. Painter, and G. Agarwal, Opt. Express 15, 16604 (2007). 